\section{REGENTES DISPOSITIONS}

\author{
CONCERNANT LA
}

\section{Législation de l'Énergie Hydroélectrique \\ (suITE)}

\section{Le Président de la République française,}

Sur le rapport du ministre des travaux publics et du ministre de l'agriculture,

Vu la loi du 16 octobre 5919 , relative à l'utilisation de l'énergie hydraulique, et en particulier l'article 28 , portant que des reglements d'administration publique détermineront notamment:

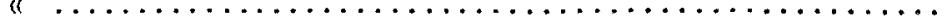

" $3^{\circ}$ Le texte des cahiers des charges type des entreprises concédées ") ;

Vu l'article 32 de la loi susvisée et notamment le paragraphe $x^{\circ r}$ ainsi conçu :

"Les décrets portant règlement d'administration publique, les décrets approuvant une concession ou accordant une autorisation ainsi que tous autres pris cn application de la présente loi, scront rendus sur le rapport et le contreseing du ministre des travaux publics. Los décrets portant règlement d'administration publiquo el les décrets approuvant une concession sur les cours d'eau no faisant pas partic du domaine public seront, en outre, contresignés par lo ministre de l'agriculture »;

$\mathrm{Vu}$ l'avis du comité consultatif des forces hydrauliques en date du 8 juillet 1920 ;

Le conseil d'Etal entendu,

\section{Décrète :}

Art. $x^{\text {er }}$ - - Est approuvé le cahier des charges lype cirannexí, dresé on exécution de l'article $2 S$ de la loi dn 16 octolore 1919 , pour les concessions de forces hydrauliques sur les cours deati et les lacs.

Art. 2. - Le ministre des travaux publics et le ministre de l'agriculture sont chargés, chacun en ce qui concerne, de Pexécution du présent décret, qui sera publić au Journal officiel de la République française et inséré au Bullelin des lois.

Fait à llambouillet, le 5 scptembre Igso.

Par le Président de la Républiquo :

P. Deschanet.

Le ministre des travaux publics,

Yves Le Thoceutr.

Le munistre des régions libérées, ministre de l'agriculhure par intérion, E. OGier.

\section{C.IIIIER IHES CIIARGES TYPE:}

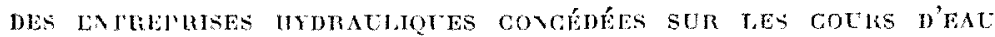
ET LES LACS

Nom de la rivière ou da fluve.

Nom al adresse du concessiomnaire.

Concession de la chute de........ comprise entre....... et......... département de..........).

\section{CAIIER DES CFARGES}

\section{CIIAPITRE PREMIIR}

OBJET DE LA CONCESSIOY

Art, I"r - Sorvice concédé. - La concession à laqualle sappli. que le présent cahier des charges a pour oljet l'établissement et l'exploitation dos ouvrages hydrauliques et de l'usine génćatrice destinćs à l'utilisation de la chute d'environ.... mètres (cn caux mojemnes) castant sur le (nom du cours dean) entre....... et...... commune de...... département dc........

La puissance maximum brute de la chute concédée est évaluéc a........ kilowats, ce qui correspond, comple tem du rende. ment normal des appareils diutilisation, à une puis ame disponible de....... kilowatts.

La puissance normale brute est évaluéc a....... hilowatls, re qui correspond de mome a the puissine normale tisponilute de....... kilowatts.

L'entrcprise a pour objot principal (3) :

Art 2. - Constslance de la concession. - Seront romsilkrés comme dépendances immolilières de la concession tous les umragos utilisés pour l’aménapement et la production de la furee hydraulique devant faire retour gratuitement à l'ktat on fin de concession, et notamment le barrage de retenue, les omranes demmagasinement, les terrains submergés, les ourriges de prise d'eau, canalisations, ouvrages régulateurs on de dicharrá, les moteurs hydrauliques (turbines et accessoires), ainsi que les terrains qui les supportent on y donnent acces et les hiliments on partie de bâtiments qui les abritent et les terrains submergés sils apparticmnent au concessionnaire ( $)$.

\section{CHAPITLE: II}

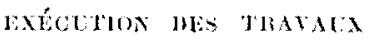

Art. 3. - Acquistlon des lorains el établissments des onortges. - Le concessionnaire sera tenu dótablir tous les ommates uliles pour l'aménagement de la fore tyodralipue ef l'exploitition de la concession ainsi que les machines et luntillage mécesaires à cet effet.

Il devra acquérir tous les termins sur lesquels seront dialilies l'usinc et ses dépendinces immobilières.

En ce qui concene l'oconpation des terains compuris dins fo

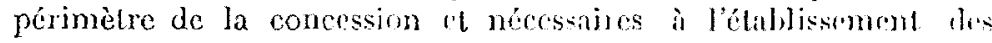
ouvrages de retenue on de prise d'eau et des canaux d'addution ou de fuite, soulerrains ou ì ciel ouvert, de mêne que puur lis terrains submergés par le relevement du plan d'ean, le conecsunnnaire bénéficiera des droils prérus à l'article 4 de la lui da I 8 octobre igrg.

Au cas ou il se bornerait ì acquérir des droils róh, molamment des scritudes d'appui, de passage on de submersion, lis contrats relatifs seront communiqqués à l'administralion de der ront comporter une dause reservant expressément a l'bial ha fatulté de se sulstituer au conersionnaire aux mémes conditions on ras de rachat ou de déchéance on ì l'expiration de la coneresion.

En outre, s'il s'agit d'une usine de plus de 10.000 kilowills, lo concessionnaire pourta oceujer temporairement fous lerrains at extraire tous matérianx nécessaircs a l'exécution das trajuan ch sc conformant aux prescriptions de la loi du 29 décembro s.jo".

Le concessionnaire pourra oceuper, dans les condltons fixres par le service compétent, sans payement de redevance spiriale, les parties du domaine fluvial néceseaires it ses inctallations.

Art. h. - Acquisition des droils à l'usage de lean. - Ponr l'accuisition des droits à l'usige de l'eaur cxercés el existaul it la date de laffichage de la demande de conerssion, le conmunim-

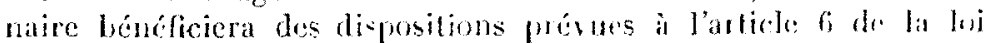
dn 16 octobre igro.

Les contrats y relatifs deronl comporter nue clause bervant. expressément ì l'Wtat la finculté de se substituer an roncesionnaire aux memes conditions on cas de rachat ou de déhíance ou à l'expiration de la concession.

Les contrats passés avec les riverains seront portés à la connaissance de lingénieur en chef, par les soins du concesiommaire

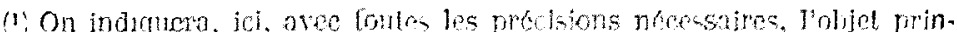

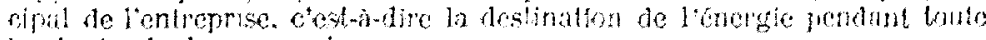
la dure de la concession.

${ }^{2}$ On spociliert evontudtement st hancession comprend egaloment los maisons de garde, batjments d'explojtalion, de. 
duns le délai dun moiś à compler de leur signature. Il en sera de mème des décisions de justice rendues par application de l'article 6 de la loi du 16 octobre 1919 , un mois après qu'elles seront devenues definitives.

Art. 5. - Caracléristique de la prise d'cal. - Le barrage ou la prise d'eau sera placé aux abords de.......

Lo niveau normal (1) de la retenue sera à la cote de....... du N. G. F,

le débit maximum cmprunté sera de........ par seconde.

Le débit maintenu dans la rivière en aval de la prise d'eau ne devra pas etre inférieur à....... par seconde.

Les caux seront restituées a......... environ.

Art. 6. - Ouvrages principaux. - Indiquer dans cet article les disposilions générules des ourrages, avec lours principales caractéristiques.

Art. 7. - Dispositions spéciales relatives d̀ la navigation, au flottage, à la circulation des poissons, cte. - Le concessionnaire sera tenu, pour faire face aux besoins de la navigation et du flottage (2):

Pour compenser les difficultés que la présence du barrage apportera aux migrations du poisson et le dépeuplement qui peut en être la conséquence, le concessionnaire fournira chaque année aux époques et sur les points indiqués par le senice compétent des alevins dont les especes et les quantités seront également indiquées par ce service, sans que toutefois la dépense correspondant à celle foumiture puisse dépasser la somme de..... fr.

Le concessionnaire sera tenu, si l'administration le recomnait nécessaire, d'élablir ef d'entretenir daus le barrage une échclle a poissons. Dans ce cas, les fournitures dalevins imposées au

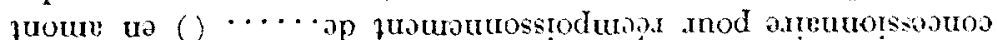
du harrage, cesseront d'ètre dues à partir de la mise en service de l'úchelle.

Le conecssionnaire pourra être tenu de placer et entretenir à lamont de la prise d'cau un grillage dont les barreaux seront espaces au maximum de..... centimetres.

Le concessionnaire devra en outre (indiquer les conditions spéciales auxquelles devront satisfaire les ourrages notamment en ce qui concerne la protection contre les inondations, la préservation des sites et paysages, la peche).

Le concessionnaire sera tenu de laisser libre circulation sur les dépendances de la concession, aux agents chargés du contrôle de la pêche.

Art. 8. - Approbation des projets. - L'exécution de tous les ouvrages dépendant de la concession, devra etre autorisée dans les formes prévues par le décret du.....

Devront ètre approuvés par le ministre des travaux publies (i)...

L'établissement des machines et l'acquisition de l'outillage pourront être effectués par le concersionnaire sans autorisation préalable s'ils proviennent de sociétés ou constructeurs français et sils ont été fabriqués en France.

Si le concessionnaire se trowe dans l'impossibilité de se procurer, en France, le matériel hydraulique et électrique, dans des conditions normales satisfaisantes de temps, de prix et de qualité, il pourra l'acquérir à l'étranger avec l'autorisation du ministre des travaux publies. Dans tous les cas, il en sera donné avis au service du contrôle.

L'approbation ou le défaut dapprobation administrative n'aura pour effet ni d'engager la responsabilité de l'administration, ni de dégager celle du concessionnaire des conséquences que pourricent avoir lexécution des travaux, l'imperfection des dispositions privucs ou le fonctionnement des ouvragres.

Art. n. - Délais dexéculion et réception des ouvrages. - Les

(1) I.e nucan normal ext. soit le nivenu legal, s'il y a des ourages lefulatems. sont lo nuvan avant le deversement.

(2) Indquer. s'il y a her les combums spectales munuelles devront

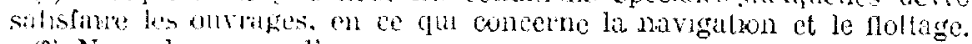

(3) Non du cours d'enu.

(1) Indiquer les ouvrages spectaux donl l'importance exigerait l'approluation du mistre. projets des travaux nécessaires pour l'aménagement de la furce motrice concédée deront étre présentés duns le déliti de..... mois, à dater de lacte de concession.

Les travaux seront commencés dans le délai de..... mois, à dater de l'approbation des projets et poursuivis sans interruption, de telle sorte qu'ils soicnt acherés et que l'usine soit mise en service dans lo délai de..... ans, à partir de la mêrne date, sauf le cas de force majeure dûment constaté.

Le projet de tout ouvrage imposé ultérieurement par l'administration au concessionnaire en exécution du présent cahier des charges, derra etre presenté dans le délai de six mois de l'invitation qui lui en sera faite, sauf dérogation justifiée par l'importance du travail, et réalisé le plus promptenent possible dans le délai fixé.

Anssitôt après l'achèvement des travaux ot, au plus tard, à l'cxpiration des délais prévus au paragraphe précédent, il sera procédé par les soins des agents du contrôle à une réception des travaux dans les formes prévues par le décrel du....... Sur le vu du procès-verbal de cette réception, le préfet autorisera, sil y a lieu, la mise en service de l'usine.

Art. Io. - Exécution el entrelien des ouvrages. - Les ouvrages, les machines et loutillage élablis en vertu de la présente concession, seront cxévutés en matériaux de bonne qualité, mis en ouvre suivant les règles de l'art et entretenus en parfait état par les soins du concessionmaire et à ses frais.

Les réparations des ouvrages resteront soumises an contròle de l'administration, qui pourra, après me mise en deneure restéc sans effet, y pourvoir d'office aux frais du concessionnaire.

Art. II. - Bomage. - Dans l'année qui suivra la mise en exploitation de l'usine, il sera procédé, aux fais du concessionnaire et au besoin d'office, au bornage des terrains fitisant partic des dépendances immobilières de la concession, contradictoirtment avec les propriétaires roisins, en présence de l'ingénieur ordinaire du service compétent, qui en dressera le procès-verbal. II sera établi aux frais du concessionnaire et sous la surveillance de l'ingénieur, un plan au ( ${ }^{2}$...... des termins ainsi borneis.

Lorsque des modifications seront apportées aux dépendances immobilieres de la concession, il sera procédé dans les mémes conditions au bornage des terrains ajoulés ou retranchés ef à l'établissernent de leur plan dans le mois qui suivra la mise en service des ouvrages établis sur ces terrains.

Art. I2. - Rétablissement des communicalions et de l'écoulement des eaux. - Le concessionnaire sera tenu de rétablir à ses frais, suivant les dispositions approuvées par l'administration compétente, les roies de communication interecptécs par ses travaux.

Il sera tenu également de rétablir et d'assurer à ses frais le libre écoulement des caux naturelles ou artificielles dont le cours scrait détourné ou modifié par ses travaux. Dans le cas où les ouvrages de la concession feraient obstacle à ce que les canaux ou rigoles d'arrosage s'alimentent comme par le passé, il pourra notamment être tenu de rétablir leur alimentation an moyen deaux prises dans ses propres canalisations. Il derra egalement prendre les dispositions qui seraient reconnues nécessaires par ladministration pour empecher que les infiltrations d caul qui proviendraient de ses canalisations nuisent aux parties basses du territoire (").

Art. I3. - Reconstulution de la produclion agricole en cas d'ćtablissemenl de grands barrages réservoirs noyant une surface imporlante de lorres cullivées. - Le conecssiomaire sera tenu de contribuer à la reconstitution de la production agricole réduite du fail de ses travaux dans les condilions suivantes $\left(^{3}\right)$ :

(1) On indiquera l'échelle qui sera en prinoipe celle de $1 / 10000$.

(2) On indiquera, sel, los conditions à umposer expressument pour lapplication des dispositions procedentes.

(3) On indaquera, jol, le concom's que le concessionmaire sera appelé a fournir sous forme de tiavaux ou de coulribution financiere aux opera. tions nécessaires pour reconstituer la production agricole redute du fat de ses travaux. 


\section{CHAPITRE III}

\section{ExPLOITATION}

Art. r/1. - Obligation de se conformer aux règlements. - Le concessionnaire sera tenu de se conformer aux règlements existants ou à intervenir, notamment en ce qui concerne la police des caux, la navigation et le flottage, la défense nationale, Ja protection contre les inondations, la salubrité publique, l'alimentation des populations riveraines, l'irrigation, la conservation et la libre circulation des poissons, la protection des sites et paysages.

Art. 15. - Obligations relalives à l'écoulement des eaux. L'administration se réserve expressément le droit de réglementer les éclusées de l'usine, en obligcant, s'il y a lieu, lo concessionnaire à maintenir, dans le canal de fuite, par un bassin de compensation ou par tous les autres dispositifs appropriés, le débit nécessaire pour sauvegarder les intérêts généraux et au besoin un débit égal à colui qui arrive à la prise d'eau, sans qu'il puisse y fairc opposition ou prétendre à une indemnité de ce chef.

Art. I6. - Obligations relatives ì l'exercice de la navigation et du fottage et à la sauvegarde des intérêts généraux. - On inscrira ici les mesures d'exploitation nécessaires a la sauvegarde des intérêts généraux (1) et notamment, sur les cours d'eau navigables ou flottables, celles propres à concilier les besoins de la navigation et du fottage avec ceux de la marche de l'usine.

Art. 17. - Obligations relatives au rejet des eaux. - Los caux empruntées seront rendues à la rivière pures, salubres et à une température voisine de cellc du bicf alimentaire.

Art. i 8. - Obligation de participer aux cntentes. - Pour l'exécution des travaux intéressant la vallée du... ou le bassin du ( 9 ...

\section{CHAPITRE IV}

\section{VENTE DE L'ÉNERGIE AU PUBLIC (3)}

Art. 19. - Tarif maximum. - Les prix auxquels le concessionnaire est autorisé à vendre l'énergie au public ne pourront pas dépasser les maxima suivants pour le courant pris a la sortie do l'usinc, sous la forme et la tension résultant du régime de ses machines génératrices ou de ses transformateurs.

Ces maxima comprennent les deux éléments suivants :

$1^{\circ}$ Une somme fixe de....... fr. par an et par kilowatt de puiscanco souscrite ;

$2^{\circ}$ Une redevance proportionnelle de..... par kilowatt-heure mesuré et livré à la sortie de l'usine génératrice.

Le concessionnaire ne sera pas tenu de fournir une puissance inféricure ì...... kilowatts.

Les tarifs maxima pourront être rúvisés tous les dix ans, soit sur la demande du concessionnaire, soil sur l'initiative de l'administration et suivant les formes adopteces pour l'approbation du présent cahier des charges.

Art. 2o. - Obligation de fournir le courant. - Lo concessionnaire sera tenu de fournir l'énergie demandée dans la limite de la puissance dont il disposera aux différents états du cours d'eatu, après avoir rëservé celle dont il a besoin pour salisfaire aux contrats dójà passés et au service de concession de distribution d'ínergic ou autres entreprises qu'il assurerait pour son compte dans les conditions déterminées par l'article $\mathrm{I}^{\text {er }}$ du cahier des charges. Au cas où les demandes d'énergie dépasseraient les disponibilités du concessionnaire, il y serait fait dro't dans l'ordre do leur inscription sur un registre spécial tenu à cet effet.

(1) On se preoccupera, en particulier, sur les rivieres à fond mobile, des mesures à prendre pour éviter les dangers risultant des affoulle. ments, exhaussements du lit et apports solides.

(2) Indiquer, ici, an cas oì un irnvall d'interat collects de la catégrie de ceux que vise l'article $28-12^{*}$ de la lol a 16 octobre 1919 serait projete dans la valle ou le bassin. les condilions spcciales dans lesquelles be concessionnaire sera tonu d'y participer.

(3) Ce chapitre ne deva pas figuror dans le camcr des charges s'il est constant que le concossionnare ne fera pas fle vente de Ínergie au public pendant touta la clurée de la concession.
Dans ces limitos, le concessionnaire sera tenu, avant l'expiration du délai d'un mois, à partir de la demande qui lui en sera faite, de fournir l'énergie électique aux conditions prévues par le cahier des charges, à toute personne qui demandera à contracter un abonnement pour une durée d'au moins...... ans. Lorsque la puissance demandée excédera...... kilowalts, le concessionnaire pourra exiger que le demandeur lui garantisse pendant..... années une recette brute annuelle de..... francs par kilowatt demandé.

Si la fourniture exige des travaux complémentaires a l'usine, le délai d'un mois prévu pour la fourniture du courant sera prolongé du temps nécossaire à l'exécution de ces travaux.

\section{CHAPITRE V}

RÉSERVES EX RUU ET EN FOHCE

Art. 2r. - Réserves en eau. - Les reserves en cau que lo concessionnaire mettra à la diaposition des services publics do l'Btat, des départements, des communes, des établissements publics ou des associations syndical's autorisces et des groupements agricoles diutilité ginérale qui seront spécifiés par un règlement d'administration publique seront fournies dans les conditions suivantes : (1)

Art. 22. - Réserves en force al profit des services publics. La puissance totale instantanée que le concessionnaire mettra, aux bornes de l'usine, i la disposition des services publics de l'Etat, des départements, des communes, des établissements publics ou des associations syndicales autorisées et des groupements agricoles d'utilitẻ générale, qui seront spécifiés dans un règlement d'administration publique, sera au maximum de $(\mathcal{Q})$.

Pendant les deux premières années ì compter de l'achèvemen'. des travaux, les demandes des services publics ou des associations susvisées devront etre satisfaites par lo concessionnaire quinze

Passé ce délai, et jusqu'ì l'expiration de la dixiòme année, à compter de l'achèvement des trivaux, le concessionnaire ne sera tenu de satisfaire à la réquisition qu'après un préavis de six mois.

Au-dela de la dixième année, et jusqu'à l'expiration de la concession, le préavis sera de douze mois.

Toute réquisition du ministre des travaux publicg faite par application du présent article pendant les cinq premières années, a compter de l'achevement des travaux, dovra etre accucillie par le concessionnaire dans les limites indiquécs ci-dessus quelle quo soit la puissance déja vendue ou employée par lui.

Dans le cas où la puissance réservée ne serait pas utiliséc en totalité à l'expiration de la cinquième annén, le pouvoir do réquisition du ministre ne pourra porter, dans les conditions indiquées ci-dessus, que sur les quantités ci-après :

Entre la cinquième et la dixième année, sur la moitié de la puissance réservée non utiliscie à la fin de la cinquième anné;

Entre la dixieme et la quinzième annce sur le tiers de la puissance réservée non utilisée à la fin de la dixième annéo;

A partir de la quinzìme année, sur le quart de la puissance réservíc non utilisce à la fin de la quinzième annće.

Toutefois, celte quantití ne pouma descendre au-dessous do...

(A suivere)

(2) Indiquer, ici, les réserves en call of les condilions de leur livmison. Spectier, $s^{\circ}$ il y a lieu, les Iravaux qui, conformement aux dispositions de l'article $10\left(6^{\circ}\right)$ de la lo1 du 16 oclobre 1019 scraient imposés au conces. sionnaire pour l'utilisation de ces reserves.

(2) Cette puissance peut tre cvaluce soit en un nombre coneret de jours après qu'clles auront été notifiées par le ministro des travaux publics.

kilowatts, nombre qui pourra, d'alleurs, varier suivant les epoques de l'année, soit en p. 100 de la puissance disponible aux divers états du cours d'eav.

Dans le calcul de la puissance, on tiendra compla, s'il y a lien, do l'énergie qui sora livréc sots forme d'eau, a prendre dans le remous du barrage ou dans le canal l'amenie.

Il est rappelé que, conformément aux disposilions de l'article $10\left(7^{\circ}\right)$ de la loi du 16 octobre 1910. ces reserves, jointes a celles de l'article 24, ne pourront priver lusme te jhus du guart de l'ónergie dont elle dispors. aux divers elats du cours d'eau. 